\title{
Health-Related Quality of Life Components in Children With Neonatal Brachial Plexus Palsy: A Qualitative Study
}

\author{
Kate W.-C. Chang, MA, MS, Amy Austin, BS, Jan Yeaman, PhD, Lauren Phillips, BS, \\ Anna Kratz, PhD, Lynda J.-S. Yang, MD, PhD, Noelle E. Carlozzi, PhD
}

\begin{abstract}
Background: Currently, no published, validated patient-reported outcome (PRO) measures of health-related quality of life (HRQOL) exist for use with neonatal brachial plexus palsy (NBPP). NBPP is a debilitating condition that occurs during the perinatal period, resulting in paralysis/paresis and loss of sensation in the affected arm. Commonly used NBPP measures are not comprehensive and do not fully account for clinically meaningful changes in function or progression of the disorder.

Objective: To evaluate important components of HRQOL for children with NBPP and identify where new PRO measures are needed.

Design: Eleven focus groups comprising children with NBPP (4), family members (6), and professional providers (1) to assess HRQOL.

Setting: Brachial plexus clinic.

Participants: Children with NBPP, their parents, and professional providers.

Inclusion Criteria: Children 7-17 years old with NBPP; parents/caregivers at least 18 years of age; professionals with $\geq 2$ years' experience providing NBPP clinical care; ability to read and speak English fluently.

Methods: Focus group sessions were recorded, transcribed verbatim, and deidentified. Qualitative frequency analysis identified different aspects of HRQOL relevant to NBPP. This analysis expands on the grounded-theory approach to qualitative analysis, including development of a domain framework, open and axial coding, selective coding, and descriptive analysis. The resulting HRQOL domain framework (and frequency analysis) was then compared to the domain framework for existing PRO measures (PROMIS and Neuro-QoL) to identify components of HRQOL where new PRO measures are needed for NBPP.

Main Outcome Measures: Not applicable.

Results: Although many physical, social, and emotional health domains were captured by existing PRO measures, some significant NBPP-specific topics emerged from qualitative analysis-functionality, sensory, physical appearance, arm/hand compensation and preference, explaining functionality/appearance to others, and self-esteem and body image concerns.

Conclusions: Development of sensitive and specific measures capturing arm/hand function and body image would improve the clinical care of patients with NBPP.

Level of Evidence: Not applicable.
\end{abstract}

\section{Introduction}

Neonatal brachial plexus palsy (NBPP) affects approximately 1.5 per 1000 live births annually in the United States $[1,2]$. This condition involves stretching of the brachial plexus nerves during the perinatal period, affecting muscle movements of the upper extremity. Although $60 \%-90 \%$ of infants with NBPP recover spontaneously at an early age, the remaining patients experience long-term residual physical impairments, including paralysis/paresis and loss of sensation in shoulder, elbow, wrist, and/or hand $[1,3,4]$. Such impairments are associated with annual medical and social costs ranging from 1 to 3 billion U.S. dollars [5].

For children with residual upper extremity impairment, customized occupational/physical therapies or surgical treatments often are recommended for regaining upper extremity function; however, treatment outcomes vary depending on palsy extension and involvement [6-9]. The uncertainty of treatment outcomes and bilateral 
discrepancies in physical appearance might contribute to associated psychosocial difficulties in children with NBPP. For example, appearance discrepancies such as muscle atrophy or arm length differences could limit participation in sports/school activities and might isolate children with NBPP from their peers in social settings [10-12].

Because of the complexity of the palsy and the fact that there are no standard guidelines for the treatment of NBPP, it is critical to understand patient expectations and overall health status to achieve maximum treatment efficacy. Patient-reported outcome (PRO) measures reflect patient and family perspectives of the physical, emotional, and social aspects of health-related quality of life (HRQOL). Currently, there are no consensus PRO measures of HRQOL for use in the NBPP population [13]. Existing measures for children with NBPP mainly focus on physical examinations such as muscle power and range of motion without indication of how these physical factors affect daily activities or psychosocial well-being. Furthermore, generic physician-developed measures such as the Child Health Questionnaire and Pediatric Outcomes Data Collection Instrument fail to capture comprehensive NBPP physical, emotional, and social concerns or NBPP-specific issues, such as sensory and body image perception [14-18]. It is critical to understand the patient's perspective of their condition to better understand the natural course of the disease, its impact on HRQOL during child development, as well as the effectiveness of interventions to improve HRQOL.

Recently, the National Institutes of Health launched the Patient-Reported Outcome Measurement Information System (PROMIS) to develop generic measures of HRQOL that are universally applicable to various conditions (http://www.nihpromis.org). Quality of Life in Neurological Disorders (Neuro-QoL) is another federally funded initiative to create, in tandem with PROMIS, measures of HRQOL that are applicable across neurologic conditions. Neuro-QoL, and PROMIS in particular, have been used widely in pediatric populations [19-26] and are designed to sensitively assess self-reported HRQOL. Although these measures have been used in several different pediatric populations, neither PROMIS nor Neuro-QoL has been examined in NBPP.

Thus, the purpose of this study was to explore HRQOL from the perspective of children with NBPP, family members, and clinicians. Specifically, we focused on identifying the relevant aspects of HRQOL that apply to individuals with NBPP, with an eye toward identifying appropriate existing PROs and areas in which new PRO development is needed for use in NBPP.

\section{Methods}

\section{Participants}

This study used a community-based partnership approach that included key NBPP stakeholders.
Specifically, we conducted 2 focus groups of children with NBPP (separate groups for ages 7-8 and 9-17), 6 groups of caregivers/parents (separate groups for parents of children ages $0-4,5-8$, and 9-17), and 1 group of NBPP professionals (eg, clinicians and researchers with more than 2 years' experience working with NBPP) to determine domains and items relevant to HRQOL in individuals with NBPP. All participants were recruited through the Brachial Plexus Palsy clinic. Inclusion criteria for the various groups were as follows: (1) children from 7 to 17 years of age with NBPP; (2) parents/caregivers who were at least 18 years of age at time of participation; and (3) $\geq 2$ years' experience providing NBPP clinical care. In addition, because focus group participation requires verbal discussion of $\mathrm{HRQOL}$ and participation requires completion of existing PRO measures, children and parent/caregivers were required to read and speak English fluently. Data were collected in accordance with local institutional review boards; participants provided informed consent (and/or assent) before participation.

\section{Data Collection and Analysis}

Each focus group discussion was a total of $\sim 90 \mathrm{mi}-$ nutes in length. Two female PhD-level group moderators with extensive focus group experience used broad, open-ended questions designed to allow participants to articulate how having NBPP has affected their (or their child's) overall HRQOL; moderators were not involved in these children's treatment. Follow-up prompts included open-ended prompts to discuss physical, social, and emotional health. Group discussion ( $\sim 60$ minutes) was then followed by the completion of several existing HRQOL measures (PROMIS/Neuro-QOL, 20 minutes) and a brief discussion of the strengths and weaknesses of these measures ( $\sim 10$ minutes). Discussions were audio-recorded, transcribed verbatim, and deidentified. Qualitative frequency analysis was conducted according to established methodology [27]. Two PhD-level investigators created physical, emotional, and social codebooks for focus group transcript coding. These codebooks were created with the World Health Organization's conceptual framework for quality of life, which includes physical, emotional, and social domains [28]. A list of subdomains was created through a detailed review of the transcripts and was revised collaboratively with the use of input from the study team until a consensus was reached.

Coding was conducted by researchers with either a Bachelor's or Master's degree and supervised by a PhDlevel psychologist. Before coding, researchers were required to establish interrater reliability $(\geq 80 \%$ agreement), to establish that they were applying the codebook similarly when coding transcripts. Once interrater reliability was established, the 2 raters were required to code the first transcript together to ensure 
Table 1

Participant demographics

\begin{tabular}{llc}
\hline Variables & No. Children & No. Family Members \\
\hline Total & 13 & 31 \\
Mean age, years \pm SD & $12 \pm 4$ & $40 \pm 10$ \\
Male & $5(38 \%)$ & $6(19 \%)$ \\
Female & $8(62 \%)$ & $25(81 \%)$ \\
Ethnicity & $11(85 \%)$ & $23(74 \%)$ \\
$\quad$ White, non-Hispanic & $0(0 \%)$ & $2(6 \%)$ \\
White, Hispanic & $2(15 \%)$ & $6(19 \%)$ \\
$\quad$ African American & & \\
Relationship to child & - & $23(74 \%)$ \\
$\quad$ Mother & - & $5(16 \%)$ \\
Father & - & $1(3 \%)$ \\
Grandparent & - & $2(6 \%)$ \\
Other family member & - & $5(16 \%)$ \\
Educational level & - & $20(65 \%)$ \\
High school & - & $6(19 \%)$ \\
College or some college & & $3(10 \%)$ \\
Master's and greater & - & $8(26 \%)$ \\
Household income & - & $13(42 \%)$ \\
Less than \$20,000 & - & \\
\$20,000-\$39,999 & - & \\
\$40,000-\$74,999 & & \\
\$75,000 and greater & & \\
\hline
\end{tabular}

$\mathrm{SD}=$ standard deviation.

reliability. Subsequently, the remaining transcripts were coded by individuals and all instances of disagreement were then reconciled with the consultation of a third individual when necessary. Frequency tables indicating the number of times each response was made according to each codebook were used to summarize the overall findings from these groups.

\section{Results}

The average age was 12 years (standard deviation $=4$ ) for children with NBPP and 40 years (standard deviation $=10$ ) for their caregiver/family member (Table 1); $38 \%$ of children and 19\% of family members were male, and $85 \%$ of children and $74 \%$ of family members were white, non-Hispanic. Family caregiver groups were composed of $90 \%$ parents (74\% mothers), and $9 \%$ were other family members (eg, grandparent). The sample composition was similar to other studies examining caregivers/parents of children with impairment $[10,29]$. Sixteen percent of the caregivers had a high school education (84\% had at least some college and/or a college or graduate degree), and $42 \%$ had family income $\$ 75,000$ and greater. The clinician group included neurosurgeons $(11 \%)$, physiatrists $(22 \%)$, plastic surgeons $(11 \%)$, nurses $(11 \%)$, social workers $(11 \%)$, clinical coordinators (11\%), and occupational therapists (22\%).

Saturation was achieved by the sixth focus group; additional groups were conducted to ensure that overall frequency counts were an accurate representation of the cohort [27]. Percentages reflect the number of times a topic was coded relative to the total number of codes, providing an index of the relative importance of each domain. Group discussion revolved around concerns with physical health $(68.9 \%$ of comments), followed by social health $(20.3 \%)$, and emotional health $(10.7 \%)$ (Table 2). A detailed breakdown of the thematic content within each domain is summarized below and presented in Table 3. The overall pattern of discussion was similar across all groups, regardless of type (child, caregiver, or provider).

\section{Physical Health}

Concerns about changes in physical health were the most frequent topic of discussion, regardless of the group composition $(68.9 \%$ of the overall discussion; Table 4). Most of this discussion focused on how the arm/hand impairment affected physical functioning and activities. Specifically, discussion included how the child's arm/hand affected functionality, physical recreational activities, activities of daily living, sensation, and lower extremity function (ie, arm/hand impairment affecting balance). There was also discussion about physical appearance of the arm/hand, techniques used to compensate for arm/hand weakness, and arm/hand preference. Across the different types of groups (child, parent/caregiver, provider), there generally was considerable agreement with regard to the relative frequency of each subdomain. Notable exceptions were that discussion within the child groups focused more on recreational activities/limitations than either the parent/caregiver or provider discussion, and clinicians discussed changes in sensation more than either the children or their parents did. Current PRO measures (PROMIS/Neuro-QoL) include content relevant to upper (arm and hand) and lower extremity (mobility and balance) function and pain; however, PROMIS/Neuro-QoL are more generic and do not focus solely on the

Table 2

HRQOL domains generated among focus groups

\begin{tabular}{|c|c|c|c|c|}
\hline \multirow[b]{2}{*}{ HRQOL Domain } & \multicolumn{4}{|l|}{ Overall Thematic Breakdown } \\
\hline & Children Focus Groups (\%) & Caregiver Focus Groups (\%) & Clinician Focus Group (\%) & Total Across All Groups (\%) \\
\hline Physical health & 61.9 & 73.8 & 62.0 & 68.9 \\
\hline Social health & 28.6 & 15.4 & 19.6 & 20.3 \\
\hline Emotional health & 9.4 & 10.2 & 16.2 & 10.7 \\
\hline
\end{tabular}

HRQOL $=$ health-related quality of life. 


\section{HRQOL Domain/Subdomain}

\section{Physical Health}

NBPP medical care

Arm/hand function

Recreation

Activities of daily living

Arm/hand sensation

Arm/hand appearance

Arm/hand compensation

Arm/hand preference

Lower extremity function

\section{Social Health}

Recreational activities

Peer relationships

Stigma

Independence

Family relationships

Explaining arm/hand to others

\section{Emotional Health}

Positive emotions

Self-esteem/body image

\section{Example Quotation}

She's been in therapy since she was 2 weeks old.

Sometimes it can be difficult because my arm gets tired after holding it up for so long.

But I mean like playing the guitar though is like a bit challenging.

It's almost to the point where she can't pick up a spoon with this hand.

You have to put the key in the ignition. So he would always have to reach around and it's very hard.

Like I have to miss my Friday lunches to go and sit with a teacher for the whole lunch and tell her what's happened. And like I have to-I'm

always getting called down to the office for another physical therapist

to come and talk to me, and I'm always like-it's like it's not-it's like both that I have to miss school work for it, number one.

It like hurts when you wake up in the morning.

I can't feel some-my left - this is numb right here.

Cause this arm's a little bit shorter than the other one, like lengthwise.

When they tell me to put my hands up I'm like arching my back to get this hand up way straight.

I would say, you know (NAME) figured out his own way of doing things about everything.

I always do everything with my right hand.

I fall down easily.

I do after school activities.

So, much of her childhood has been spent in isolation away from other children.

Like this young girl telling me Friday, the other girls are getting asked out and boys won't talk to her.

She told me to stop using my arm because she actually did say it looks awkward flopping around like that.

I've had four year olds tell me the other kids don't want to play with them cause they have a dumb arm.

I don't like people doing stuff for me at all.

She would spend all day trying to do one thing so she could do it herself, you know.

My family is really helpful.

When they ask what's wrong with her arm, she always has the right answers.

It's hard for fitting in. Like kids will make remarks. Like what's that thing? Why is it like that?

It made her a little more determined.

He kept referring to it as a stupid arm.

Overall Thematic Breakdown

Children Focus Caregiver Focus Clinician Focus Total Across All

Groups (\%) $\quad$ Groups (\%)

Group (\%)

Total Across Al

$\begin{array}{llll}61.9 & 73.8 & 62.0 & 68.9\end{array}$

$\begin{array}{llll}61.9 & 73.8 & 62.0 & 68.9 \\ 24.4 & 35.3 & 12.0 & 29.8\end{array}$

17.4

19.8

19.9

29.7

6.3

12.4

9.5

19.2

$\begin{array}{ll}4.8 & 22.2\end{array}$


hand/arm impairment that is characteristic of NBPP (although they do capture some aspects of arm/hand function, activities of daily living, and pain). For example, NBPP-specific concerns such as differences in physical appearance (eg, with regard to the arm length difference), functional compensation (using their affected arm in unique ways to complete functional tasks), and arm/hand preference are not captured by existing measures. In addition, existing measures do not adequately capture NBPP-specific aspects of medical care/adherence, recreation, appearance, compensatory approaches, and preference.

\section{Social Health}

The next most frequently discussed topic was how NBPP affected social health $(20.3 \%$ of the overall discussion; Table 4). Discussion included recreational activities, peer and family relationships, stigma (and explaining the arm/hand to others), and being able to do things independently. Again, the pattern of discussion across the different types of groups (child, parent/ caregiver, and provider) generally was similar. There were 2 notable exceptions: (1) parents and providers discussed issues of stigma more frequently than children, and (2) parents focused on independence of their child more often than either the children themselves or the providers did. Although PROMIS/Neuro-QoL includes measures of peer relationships, stigma, and family relationships, topics such as recreation, independence, and having to explaining hand/arm differences are not currently captured by existing PROs.

\section{Emotional Health}

Finally, the least frequent topic of discussion across all groups was emotional health $(10.7 \%$ of the overall discussion; Table 4). The most commonly discussed aspect of emotional health was positive psychological functioning for both children and their parents/caregivers. Discussion focused on how the impairment improved their lives, their resilience, and their determination. Self-esteem and body image also were very important points of discussion, especially for parent/ caregivers and providers. Although children rarely discussed body image or self-esteem, this was a topic of conversation among parents; thus, the group setting for children may have precluded discussion in this area. Anger/frustration also was discussed, but this was raised more as a concern by parents than by children or providers. Furthermore, children and providers voiced concerns with anxiety, but this was discussed with less frequency among parents/caregivers. Finally, sadness/ depression also was discussed, but less frequently than many of the other emotional health concepts. The majority of emotional concerns raised are captured within the PROMIS/Neuro-QoL framework (anxiety, depression, 
Table 4

Thematic overlap among patient-reported outcome measurements

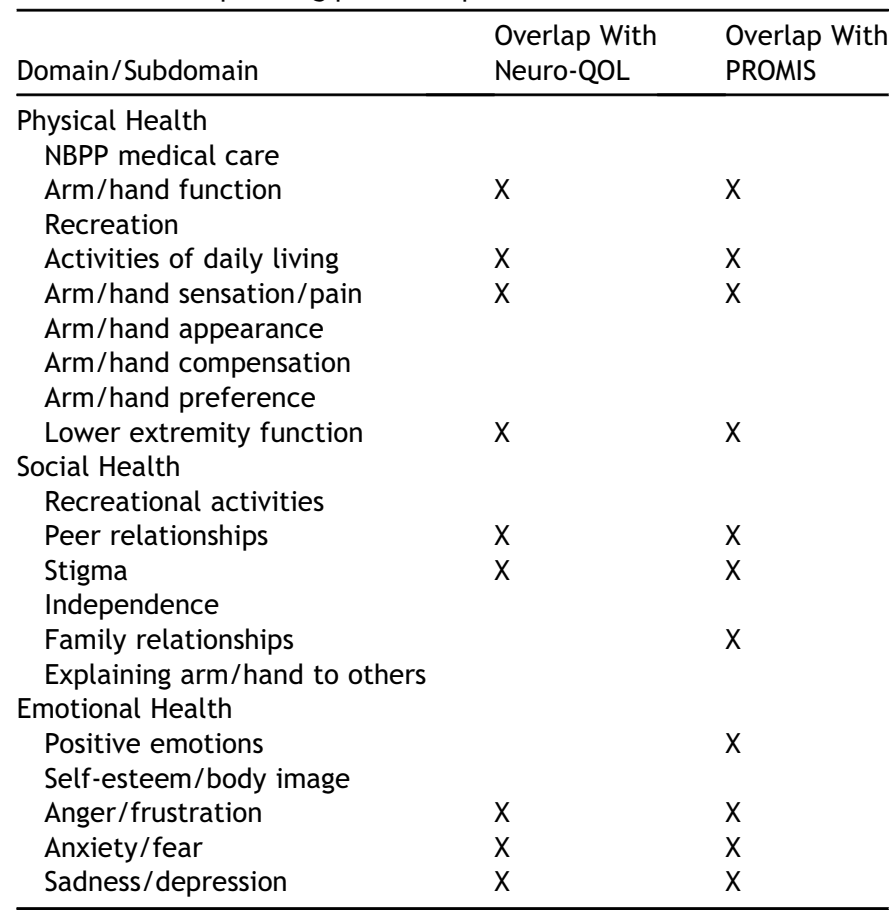

Neuro-QoL $=$ Quality of Life in Neurological Disorders; PROMIS = Patient-Reported Outcome Measurement Information System; NBPP = neonatal brachial plexus palsy.

anger, positive emotions) with the notable exception of self-esteem/body image.

\section{Discussion}

This study is the first detailed exploration of NBPPspecific HRQOL. Our findings reveal how children, family members, and clinical providers perceive NBPP and how this condition affects various aspects of a child's daily life. In addition, although there was general agreement among children, caregivers, and providers about physical, social, and emotional health, some important discrepancies existed. Furthermore, unique aspects of HRQOL (ie, those specific to NBPP and not captured by existing measurement systems) emerged.

The majority of the group discussion revolved around physical health (68.9\% across all groups); this finding was consistent with our previous studies in that physical function plays a critical role in HRQOL $[10,17,18]$. From the children's perspective, limited range of motion or strength was described as affecting their ability to perform certain movements in sports or school activities. Similar concerns regarding difficulties with physical function in daily activities were also reported in a crosssectional study of adolescents with NBPP in Sweden [12]. Topics such as compensation for lack of range of motion/ strength with different body movements, as well as a preference for using the unaffected arm to accomplish tasks, were common and specific to NBPP HRQOL. In general, children, caregivers, and providers expressed similar concerns, except that parents focused more on the NBPP medical care experience, including surgical treatment and therapies since birth. This finding may reflect the fact that parents are involved frequently in the medical decision-making process for treatment of their child's NBPP, whereas the child might not recall his or her entire medical treatment since birth [18].

The second most frequent topic of discussion was social health ( $20.3 \%$ of the discussion across all groups). Recreation, peer relationships, and stigma were reported the most frequently for all 3 group types. Because physical impairment or aesthetic differences compared with their healthy peers, children with NBPP might experience social difficulties when participating in sports or school activities [11]. Stigma is a very common social health theme among children with chronic illness. Because the majority of a child's daily living involves school activities, being isolated and excluded from group activities might affect a child's psychosocial stabilities [17,30-33]. In addition, children and adolescents tend to value support from their peers and family the most, regardless of age or gender [34]. Therefore, fostering strong peer relationships and perceived peer support could be beneficial for children with NBPP to develop positive coping strategies, reduce social difficulties, and improve their psychosocial health $[11,35,36]$.

Furthermore, schools and teachers also should be aware of the physical and social challenges that children with NBPP might encounter in the school setting. Programs or educational materials to help teachers understand the unique needs of children with NBPP should be advantageous. On the other hand, family relationships could also be challenged in the treatment of children with NBPP. Time commitment and financial burdens from constant clinic visits, therapy sessions, or even surgical interventions could cause stress to caregivers. Previous studies investigating the impact of NBPP on parents and/or families showed that this condition not only affected children's HRQOL, but also influenced the whole family $[29,30,37,38]$. An interdisciplinary clinic with social work services could provide comprehensive support and resources for patients, families, and schools to help reduce the impact of social difficulties while treating children with NBPP.

Finally, participants discussed concerns over emotional health $(10.7 \%$ of the discussion across all groups). Positive psychological functioning, such as empathy, strong will, and determination, was expressed among the children and caregiver groups; this could be the result $f$ the sense of being a "survivor," similar to the pediatric cancer survivor population [20]. Growing up with NBPP, most children receive treatments without knowing whether their arm may or may not regain function. The uncertainty of NBPP outcomes might trigger negative emotions such as stress, anxiety, or 
fear. In studies of children with congenital/acquired limb deficiencies, they reported greater incidences of anxiety, depressive symptoms, and low self-esteem in children with limb impairments [36,39]. The mixedgender focus group setting may have limited an indepth discussion of self-esteem, sexuality, and dating in the children's group. Self-esteem was, however, raised as an important area of concern, especially among caregivers and providers. Associations between physical impairment and self-esteem have been established in the literature $[11,12,30,35]$. Counterintuitively, children with NBPP might not necessarily demonstrate lower self-esteem than their healthy peers, especially when participating in an activity of their choice $[11,12,30]$. Nonetheless, providing educational sessions, social support, and guidance in coping strategies could potentially enhance emotional health in children with NBPP $[10,11]$.

Although various aspects of HRQOL were included in PROMIS/Neuro-QoL measures, discrepancies exist in thematic overlap between focus group domains and existing PRO measures. PROMIS/Neuro-QoL have been used in different pediatric populations with chronic diseases [19-26]. One of the difficulties when applying PROMIS/Neuro-QoL to populations with chronic illnesses is that current PRO measures might be too generic to distinguish HRQOL differences between disease level as well as $\mathrm{HRQOL}$ changes during treatment. Although a recently published study [20] demonstrated how PROMIS could be used across 6 common pediatric chronic diseases (asthma, cancer, chronic kidney disease, obesity, rheumatic disease, and sickle cell disease), our study results suggest that PROMIS/Neuro-QoL might not be comprehensive enough to capture the NBPP-specific HRQOL concerns, especially with regard to arm/hand functioning. Specifically, NBPP-specific domains including $\mathrm{arm} / \mathrm{hand}$ appearance, compensation, preference, independence, and self-esteem/body image are not currently assessed in these existing systems and are critical aspects of successful clinical outcomes in these children. Thus, a combination of these existing measures and new measures that capture the important NBPP-specific aspects of HRQOL is warranted.

While this study is among the first to focus on patientreported HRQOL in NBPP, it is important to recognize several limitations. The qualitative setting of focus groups provided an opportunity for participants to express their opinions freely; however, recall bias might exist and the collected data might be subject to investigator bias $[40,41]$. To eliminate bias, the investigators who conducted the focus group discussions were not involved in participants' medical care. During data analysis, 2 independent coders reconciled and reached interrater reliability ( $\geq 80 \%$ agreement) for transcript coding. This method could ensure the consistency and comprehensiveness of the data analysis. The focus group setting among peers could limit discussion on "personal" topics that might emerge; future studies could apply a one-on-one interview setting to collect more in-depth data, particularly on sensitive topics. In addition, focus group moderators were women, which may have influenced the focus group dynamic, especially for the adolescent boys in our groups. Future work might consider using a semistructured interview approach that includes same-sex interviewer/participant dyads.

Furthermore, we used a qualitative frequency approach designed to quantify discussion topics; this can be contrasted with more traditional narrative qualitative analysis approaches, and thus, may not yield the same pattern of findings. Also, our limited sample size and the limited number of fathers participating in these groups potentially limited the generalizability of these findings to the broader NBPP populations. In addition, our focus on children and parents/caregivers who were fluent in English precludes our ability to generalize these results to individuals who are not fluent in English. Our sample consisted mostly of non-Hispanic white individuals, which may limit generalizability to a broader range of races, ethnicities, and cultures. Lastly, although our provider focus group comprised a range of professionals that participate in an interdisciplinary NBPP-focused clinic, not all types of providers that provide care to patients with NBPP were included; for instance, the group did not include a neurologist or physical therapist (although the physiatrist in the group oversees physical therapy). Nevertheless, this qualitative study aimed to explore NBPP-specific PROs that were not captured by PROMIS/ Neuro-QoL, and provides the foundation for future studies.

\section{Conclusion}

Our study results revealed that HRQOL is a multifaceted and important construct for individuals with NBPP. NBPP-specific HRQOL domains such as medical care, recreation, arm/hand appearance, compensation and preference, independence, having to explain the arm/ hand to others, and self-esteem/body image emerged during the study. In addition, more generic domains, such as arm/hand function, sensation/pain, activities of daily living, peer relationships, stigma, anger/frustration, anxiety/fear, and sadness/depression from existing measurement systems (PROMIS/Neuro-QoL) include content that is relevant to NBPP. In order to provide comprehensive care for patients with NBPP, PROs are needed that include NBPP-specific content to better understand overall HRQOL in children with NBPP.

\section{References}

1. Pondaag W, Malessy MJ, van Dijk JG, Thomeer RT. Natural history of obstetric brachial plexus palsy: A systematic review. Dev Med Child Neurol 2004;46:138-144. 
2. Sheffler LC, Lattanza L, Hagar Y, Bagley A, James MA. The prevalence, rate of progression, and treatment of elbow flexion contracture in children with brachial plexus birth palsy. J Bone Joint Surg Am 2012;94:403-409.

3. Hoeksma AF, ter Steeg AM, Nelissen RG, van Ouwerkerk WJ, Lankhorst GJ, de Jong BA. Neurological recovery in obstetric brachial plexus injuries: An historical cohort study. Dev Med Child Neurol 2004;46:76-83.

4. Waters PM. Comparison of the natural history, the outcome of microsurgical repair, and the outcome of operative reconstruction in brachial plexus birth palsy. J Bone Joint Surg Am 1999;81: 649-659.

5. Chauhan SP, Blackwell SB, Ananth CV. Neonatal brachial plexus palsy: Incidence, prevalence, and temporal trends. Semin Perinatol 2014;38:210-218.

6. Hale HB, Bae DS, Waters PM. Current concepts in the management of brachial plexus birth palsy. J Hand Surg Am 2010;35:322-331.

7. McNeely PD, Drake JM. A systematic review of brachial plexus surgery for birth-related brachial plexus injury. Pediatr Neurosurg 2003;38:57-62.

8. Squitieri L, Steggerda J, Yang LJ, Kim HM, Chung KC. A national study to evaluate trends in the utilization of nerve reconstruction for treatment of neonatal brachial plexus palsy [outcomes article]. Plast Reconstr Surg 2011;127:277-283.

9. Terzis JK, Kokkalis ZT. Pediatric brachial plexus reconstruction. Plast Reconstr Surg 2009;124:e370-e385.

10. Franzblau LE, Chung KC, Carlozzi N, Chin AY, Nellans KW, Waljee JF. Coping with congenital hand differences. Plast Reconstr Surg 2015;135:9.

11. Mentrikoski JM, Duncan CL, Melanson A, et al. Factors related to the psychosocial functioning of youth with neonatal brachial plexus injuries. J Pediatr Psychol 2015;40:285-296.

12. Strombeck C, Fernell E. Aspects of activities and participation in daily life related to body structure and function in adolescents with obstetrical brachial plexus palsy: A descriptive follow-up study. Acta Paediatr 2003;92:740-746.

13. Chang KW, Justice D, Chung KC, Yang LJ. A systematic review of evaluation methods for neonatal brachial plexus palsy. J Neurosurg Pediatr 2013;12:395-405.

14. Bae DS, Waters PM, Zurakowski D. Correlation of pediatric outcomes data collection instrument with measures of active movement in children with brachial plexus birth palsy. J Pediatr Orthop 2008;28:584-592.

15. Dedini RD, Bagley AM, Molitor F, James MA. Comparison of pediatric outcomes data collection instrument scores and range of motion before and after shoulder tendon transfers for children with brachial plexus birth palsy. J Pediatr Orthop 2008;28:259-264.

16. Nath RK, Avila MB, Karicherla P, Somasundaram C. Assessment of triangle tilt surgery in children with obstetric brachial plexus injury using the pediatric outcomes data collection instrument. Open Orthop J 2011;5:385-388.

17. Squitieri L, Larson BP, Chang KW, Yang LJ, Chung KC. Understanding quality of life and patient expectations among adolescents with neonatal brachial plexus palsy: A qualitative and quantitative pilot study. J Hand Surg Am 2013;38:2387-2397.

18. Squitieri L, Larson BP, Chang KW, Yang LJ, Chung KC. Medical decision-making among adolescents with neonatal brachial plexus palsy and their families: A qualitative study. Plast Reconstr Surg 2013;131:880e-887e.

19. Adkinson JM, Bickham RS, Chung KC, Waljee JF. Do patient- and parent-reported outcomes measures for children with congenital hand differences capture WHO-ICF domains? Clin Orthop Relat Res 2015;473:3549-3563.

20. DeWalt DA, Gross HE, Gipson DS, et al. PROMIS((R)) pediatric selfreport scales distinguish subgroups of children within and across six common pediatric chronic health conditions. Qual Life Res 2015;24:2195-2208.
21. Irwin DE, Gross HE, Stucky BD, et al. Development of six PROMIS pediatrics proxy-report item banks. Health Qual Life Outcomes 2012;10:22.

22. Kratz AL, Slavin MD, Mulcahey MJ, Jette AM, Tulsky DS, Haley SM. An examination of the PROMIS pediatric instruments to assess mobility in children with cerebral palsy. Qual Life Res 2013;22: 2865-2876.

23. Mulcahey MJ, Haley SM, Slavin MD, et al. Ability of PROMIS pediatric measures to detect change in children with cerebral palsy undergoing musculoskeletal surgery. J Pediatr Orthop 2015 June 5 [Epub ahead of print].

24. Shapiro DN, Waljee J, Ranganathan K, Buchman S, Warschausky S. Using the patient reported outcomes measurement information system to evaluate psychosocial functioning among children with craniofacial anomalies. Plast Reconstr Surg 2015;135:1673-1679.

25. Varni JW, Stucky BD, Thissen D, et al. PROMIS Pediatric Pain Interference Scale: An item response theory analysis of the pediatric pain item bank. J Pain 2010;11:1109-1119.

26. Waljee JF, Carlozzi N, Franzblau LE, Zhong L, Chung KC. Applying the patient-reported outcomes measurement information system to assess upper extremity function among children with congenital hand differences. Plast Reconstr Surg 2015;136:200e-207e.

27. Kisala PA, Tulsky DS. Opportunities for CAT applications in medical rehabilitation: Development of targeted item banks. J Appl Meas 2010;11:315-330.

28. World Health Organization. Preamble to the Constitution of the World Health Organization as adopted by the International Health Conference, New York, 19-22 June, 1946; signed on 22 July 1946 by the representatives of 61 States (Official Records of the World Health Organization, no. 2, p. 100) and entered into force on 7 April 1948.

29. Louden E, Allgier A, Overton M, Welge J, Mehlman CT. The impact of pediatric brachial plexus injury on families. J Hand Surg Am 2015; $40: 1190-1195$.

30. Akel BS, Oksuz C, Oskay D, Firat T, Tarakci E, Leblebicioglu G. Health-related quality of life in children with obstetrical brachial plexus palsy. Qual Life Res 2013;22:2617-2624.

31. Boling S, Varho T, Kiviranta T, Haataja L. Quality of life of Finnish children with cerebral palsy. Disabil Rehabil 2015;38:683-688.

32. Graf A, Landolt MA, Mori AC, Boltshauser E. Quality of life and psychological adjustment in children and adolescents with neurofibromatosis type 1. J Pediatr 2006;149:348-353.

33. Joachim G, Acorn S. Stigma of visible and invisible chronic conditions. J Adv Nurs 2000;32:243-248.

34. Bokhorst CL, Sumter SR, Westenberg PM. Social support from parents, friends, classmates, and teachers in children and adolescents aged 9 to 18 years: Who is perceived as most supportive? Soc Dev 2010;19:417-426.

35. Jemta L, Fugl-Meyer KS, Oberg K, Dahl M. Self-esteem in children and adolescents with mobility impairment: Impact on well-being and coping strategies. Acta Paediatr 2009;98:567-572.

36. Varni JW, Setoguchi Y, Rappaport LR, Talbot D. Psychological adjustment and perceived social support in children with congenital/acquired limb deficiencies. J Behav Med 1992;15:31-44.

37. Firat T, Oskay D, Akel BS, Oksuz C. Impact of obstetrical brachial plexus injury on parents. Pediatr Int 2012;54:881-884.

38. Oskay D, Oksuz C, Akel S, Firat T, Leblebicioglu G. Quality of life in mothers of children with obstetrical brachial plexus palsy. Pediatr Int 2012;54:117-122.

39. Varni JW, Rubenfeld LA, Talbot D, Setoguchi Y. Determinants of self-esteem in children with congenital/acquired limb deficiencies. J Dev Behav Pediatr 1989;10:13-16.

40. Creswell JW. Research Design: Qualitative, Quantitative, and Mixed Method Approaches. Thousand Oaks, CA: SAGE Publications, Inc; 2003.

41. Silverman D, Marvasti A. Doing Qualitative Research: A Comprehensive Guide. Thousand Oaks, CA: SAGE Publications, Inc; 2008. 


\section{Disclosure}

K.W.-C.C. Department of Neurosurgery, University of Michigan, Ann Arbor, MI Disclosure: nothing to disclose

A.A. Department of Physical Medicine and Rehabilitation, University of Michigan, Ann Arbor, MI

Disclosure: nothing to disclose

J.Y. Department of Psychology, Spring Arbor University, Jackson, MI Disclosure: nothing to disclose

L.P. Department of Psychology, Spring Arbor University, Jackson, MI Disclosure: nothing to disclose

A.K. Department of Physical Medicine and Rehabilitation, University of Michigan, Ann Arbor, Ml

Disclosure: nothing to disclose
L.J.-S.Y. Department of Neurosurgery, University of Michigan, Ann Arbor, MI Disclosure: nothing to disclose

N.E.C. Department of Physical Medicine and Rehabilitation, University of Michigan, 2800 Plymouth Road, Ann Arbor, MI 48109-2800. Address correspondence to: N.E.C.; e-mail: carlozzi@med.umich.edu

Disclosure: nothing to disclose

This work was supported by MedSAU Fund.

Submitted for publication March 22, 2016; accepted August 1, 2016. 